Research Article

\title{
Strong Convergence on the Split Feasibility Problem by Mixing $W$-Mapping
}

\author{
Fugen Gao, Xiaoxiao Liu, and Xiaochun Li \\ College of Mathematics and Information Science, Henan Normal University, Xinxiang 453007, Henan, China \\ Correspondence should be addressed to Xiaochun Li; 1xiaochun07@126.com
}

Received 6 March 2021; Accepted 15 May 2021; Published 27 May 2021

Academic Editor: Kaleem R. Kazmi

Copyright (c) 2021 Fugen Gao et al. This is an open access article distributed under the Creative Commons Attribution License, which permits unrestricted use, distribution, and reproduction in any medium, provided the original work is properly cited.

In this paper, we concern with the split feasibility problem (SFP) in real Hilbert space whenever the sets involved are nonempty, closed, and convex. By mixing $W$-mapping with the viscosity, we introduce a new iterative algorithm for solving the split feasibility problem, and we prove that our proposed algorithm is convergent strongly to a solution of the split feasibility problem.

\section{Introduction}

Throughout this article, we assume that $H_{1}$ and $H_{2}$ are two real Hilbert spaces. The split feasibility problem (SFP) was introduced by Censor and Elfving [1], and it is formulated as finding a point $x$ in $C$ such that $A x$ is in $Q$, namely,

$$
\begin{array}{r}
x \in C, \\
A x \in Q,
\end{array}
$$

where $C$ and $Q$ are nonempty, closed, and convex subsets of real Hilbert spaces $H_{1}$ and $H_{2}$, respectively, and $A$ is a bounded linear operator from $H_{1}$ to $H_{2}$.

Many inverse problems arising from various fields of science and technology, such as intensity-modulated radiation therapy [2], signal processing, and image reconstruction, can be summarized as SFP. Due to its applications, many algorithms have been invented to solve SFP (see, for instance, [3-11]).

To solve problem (1), in 2002, Byren [3] introduced a popular algorithm which is called the CQ-algorithm as follows:

$$
x_{n+1}=P_{C}\left[I-\mu_{n} A^{*}\left(I-P_{Q}\right) A\right] x_{n}
$$

where $I$ is the identity operator on $H, P_{C}$ and $P_{Q}$ denote the metric projection onto the closed convex subsets $C$ and $Q$, respectively, and $A^{*}$ is the adjoint operator of $A$ and $0<\mu_{n}<\left(2 /\|A\|^{2}\right)$. In 2018, Wang [10] proposed his algorithm as follows:

$$
x_{n+1}=x_{n}-\tau_{n}\left[\left(I-P_{C_{n}}\right) x_{n}+A^{*}\left(I-P_{Q_{n}}\right) A x_{n}\right],
$$

where $\left\{C_{n}\right\},\left\{Q_{n}\right\}$, and $\left\{\tau_{n}\right\}$ were given by

$$
\begin{aligned}
C_{n} & =\left\{x \in H_{1}: c\left(x_{n}\right) \leq\left\langle\xi_{n}, x_{n}-x\right\rangle\right\}, \quad \xi_{n} \in \partial c\left(x_{n}\right), \\
Q_{n} & =\left\{y \in H_{2}: q\left(A x_{n}\right) \leq\left\langle\zeta_{n}, A x_{n}-y\right\rangle\right\}, \quad \zeta_{n} \in \partial q\left(A x_{n}\right),
\end{aligned}
$$

in which $c: H_{1} \longrightarrow R$ and $q: H_{2} \longrightarrow R$ are two given convex functions, and

$$
\tau_{n}=\lambda_{n} \frac{\left\|x_{n}-P_{C_{n}} x_{n}\right\|^{2}+\left\|\left(I-P_{Q_{n}}\right) A x_{n}\right\|^{2}}{2\left\|\left(I-P_{C_{n}}\right) x_{n}+A^{*}\left(I-P_{Q_{n}}\right) A x_{n}\right\|^{2}},
$$

in which $\lambda_{n} \in(0,4)$.

To obtain strong convergence theorem, Wang [10] modified his algorithm as follows:

$$
\begin{aligned}
y_{n} & =x_{n}-\tau_{n}\left[\left(I-P_{C_{n}}\right) x_{n}+A^{*}\left(I-P_{Q_{n}}\right) A x_{n}\right], \\
x_{n+1} & =\alpha_{n} u+\left(1-\alpha_{n}\right) y_{n},
\end{aligned}
$$

where $\left\{C_{n}\right\},\left\{Q_{n}\right\}$, and $\left\{\tau_{n}\right\}$ were given as the same to the weak convergence theorem; $\left\{\alpha_{n}\right\}$ is a sequence in $[0,1]$ which is chosen so that 


$$
\begin{aligned}
& \lim _{n \longrightarrow \infty} \alpha_{n}=0, \\
& \sum_{n=0}^{\infty} \alpha_{n}=\infty, \\
& \text { either } \sum_{n=0}^{\infty}\left|\alpha_{n+1}-\alpha_{n}\right|<\infty \\
& \text { or } \lim _{n \longrightarrow \infty}\left(\frac{\alpha_{n+1}}{\alpha_{n}}\right)=1
\end{aligned}
$$

On the other hand, another problem which is similar to the split feasibility problem is the proximal split feasibility problem (PSFP), and the proximal split feasibility problem is to find a point $x^{*}$ satisfying the property:

$$
\begin{gathered}
x^{*} \in \arg \min f, \\
A x^{*} \in \arg \min g,
\end{gathered}
$$

where $f: H_{1} \longrightarrow R \cup\{\infty\}$ and $g: H_{2} \longrightarrow R \cup\{\infty\}$ are two proper and lower semicontinuous convex functions, $A: H_{1} \longrightarrow H_{2}$ is a linear bounded operator, and $\arg \min f:=\left\{\bar{x} \in H_{1}: f(\bar{x}) \leq f(x), \forall x \in H_{1}\right\}$, arg min $g:=\left\{\bar{y} \in H_{2}: g(\bar{y}) \leq g(y), \forall y \in H_{2}\right\}$.

To solve problem (8), in 2014, Moudafi and Thakur [12] introduced the split proximal algorithm by the following iterative scheme:

$$
x_{n+1}=\operatorname{prox}_{\lambda \mu_{n} f}\left(x_{n}-\mu_{n} A^{*}\left(I-\operatorname{prox}_{\lambda g}\right) A x_{n}\right),
$$

where the stepsize $\left\{\mu_{n}\right\}$ was the suitable positive real number sequence, and they also proved the weak convergence of the sequence generated by the above equation to a solution of problem (8). In 2015, Shehu et al. [13] introduced a viscositytype algorithm for solving proximal split feasibility problems as follows:

$$
\begin{gathered}
y_{n}=x_{n}-\mu_{n} A^{*}\left(I-\operatorname{prox}_{\lambda g} A\right) x_{n}, \\
x_{n+1}=\alpha_{n} \psi\left(x_{n}\right)+\left(1-\alpha_{n}\right) \operatorname{prox}_{\lambda \mu_{n} f} y_{n},
\end{gathered}
$$

where $\psi: H_{1} \longrightarrow H_{1}$ is a contraction mapping. They also proved a strong convergence of the sequence generated by iterative scheme (10) in Hilbert spaces. Recently, Shehu and Iyiola [14] introduced the following algorithm for solving split proximal problems and fixed point problems in Hilbert spaces:

$$
\begin{aligned}
u_{n} & =\left(1-\alpha_{n}\right) x_{n}, \\
y_{n} & =\operatorname{prox}_{\lambda \gamma_{n} f}\left(u_{n}-\gamma_{n} A^{*}\left(I-\operatorname{prox}_{\lambda g}\right) A u_{n}\right), \\
x_{n+1} & =\left(1-\beta_{n}\right) y_{n}+\beta_{n} T y_{n},
\end{aligned}
$$

where $T$ is a $k$-strictly pseudocontractive mapping. They also showed that, under certain assumptions imposed on the parameters, the sequence $\left\{x_{n}\right\}$ was generated by the algorithm that they introduced converges strongly to $x^{*} \in F(T) \cap \arg \min f \cap A^{-1}(\arg \min g)$.

If we defined $f=i_{C}$ and $g=i_{Q}$ as indicated functions of sets $C$ and $Q$, where $C$ and $Q$ are nonempty, closed, and convex sets of $H_{1}$ and $H_{2}$, respectively, then the proximal split feasibility problem (8) becomes the split feasibility problem (1). In this paper, inspired and motivated by these works that have been done, we focus on the split feasibility problem in Hilbert spaces.

The rest of this paper is organized as follows. In Section 2 , we review some definitions and lemmas that we need. In Section 3, we introduce a new iterative algorithm based on the viscosity method and $W$-mapping which is defined in Section 2 for finding a solution of the split feasibility problem and prove a strong convergence theorem under some mild conditions.

\section{Preliminaries}

Throughout this paper, let $H_{i}(i=1,2)$ be a real Hilbert space with the inner product $\langle\cdot, \cdot\rangle$ and norm $\|\cdot\|$. We denote by $I$ the identity operator on $H_{i}(i=1,2)$ and by $w_{w}\left(x_{n}\right)$ the set of all weak cluster points of $\left\{x_{n}\right\}$. The notation $\longrightarrow$ stands for strong convergence and $\rightarrow$ stands for weak convergence.

Definition 1 (see [15]). Let $T: H \longrightarrow H$ be a nonlinear mapping. Then, $T$ is

(1) Nonexpansive if

$$
\|T x-T y\| \leq\|x-y\|, \quad \text { for } \forall x, y \in H_{1} .
$$

(2) Firmly nonexpansive if

$\|T x-T y\|^{2}+\|(I-T) x-(I-T) y\|^{2} \leq\|x-y\|^{2}, \quad$ for $\forall x, y \in H_{1}$.

Definition 2. Let $C$ be a nonempty closed convex subset of $H_{1}$. Then, an orthogonal projection $P_{C}: H_{1} \longrightarrow C$ is defined by

$$
P_{C} x=\underset{y \in C}{\arg \min }\|x-y\|^{2}, \quad x \in H_{1}
$$

Lemma 1. Let $C$ be a nonempty closed convex subset of $H_{1}$, then

(1) $\left\langle x-P_{C} x, z-P_{C} x\right\rangle \leq 0, \forall x \in H_{1}, z \in C$.

(2) $P_{C}$ and $I-P_{C}$ both are (firmly) nonexpansive.

(3) $\left\langle P_{C} x-P_{C} y, x-y\right\rangle \geq\left\|P_{C} x-P_{C} y\right\|^{2}, \forall x, y \in H_{1}$.

(4) $\left\langle x-z, x-P_{C} x\right\rangle \geq\left\|x-P_{C} x\right\|^{2}, \forall x \in H_{1}, z \in C$.

Definition 3. Let $T: H_{1} \longrightarrow H_{1}$ be an operator with $\operatorname{Fix}(T) \neq \varnothing$. If for any $\left\{x_{n}\right\}$ in $H_{1}, x_{n} \rightarrow x$ and $(I-T) x_{n} \longrightarrow 0$, we can obtain $x \in \operatorname{Fix}(T)$, then we claim that $I-T$ is demiclosed at zero.

Lemma 2. If $T: H_{1} \longrightarrow H_{1}$ is a nonexpansive operator, then $I-T$ is demiclosed at zero.

Lemma 3. For all $x, y \in H_{1}$, and $\eta>0$, we have 
(1) $\|x+y\|^{2} \leq\|y\|^{2}+2\langle x, x+y\rangle$.

(2) $\|x+y\|^{2} \leq(1+\eta)\|x\|^{2}+(1+(1 / \eta))\|y\|^{2}$.

Definition 4. If for any $n \geq 1$ and $z \in C$, let $S_{i}(1 \leq i \leq n)$ be a nonexpansive mapping on $C$ and $\eta_{i}$ be real numbers with $0<\eta_{i} \leq \eta<1$. We define a mapping $W_{n}$ on $C$ for each $n \geq 1$ by

$$
\begin{aligned}
& U_{n, n+1}= I \\
& U_{n, n}=\left(1-\eta_{n}\right) I+\eta_{n} S_{n} U_{n, n+1} \\
& U_{n, n-1}=\left(1-\eta_{n-1}\right) I+\eta_{n-1} S_{n-1} U_{n, n} \\
& \vdots \\
& U_{n, k}=\left(1-\eta_{k}\right) I+\eta_{k} S_{k} U_{n, k+1} \\
& U_{n, k-1}=\left(1-\eta_{k-1}\right) I+\eta_{k-1} S_{k-1} U_{n, k} \\
& \vdots \\
& U_{n, 2}=\left(1-\eta_{2}\right) I+\eta_{2} S_{2} U_{n, 3} \\
& U_{n, 1}=\left(1-\eta_{1}\right) I+\eta_{1} S_{1} U_{n, 2} \\
& W_{n}=U_{n, 1},
\end{aligned}
$$

then we say that $W_{n}$ is the $W$-mapping generated by $S_{n}, S_{n-1}, \ldots, S_{1}$ and $\eta_{n}, \eta_{n-1}, \ldots, \eta_{1}$.

Lemma 4 (see [16]). Let $W_{n}: H_{1} \longrightarrow H_{1}$ be a $W$-mapping, then $W_{n}$ is a nonexpansive mapping.

Lemma 5 (see [17]). Assume that $\left\{s_{k}\right\}$ is a sequence of nonnegative real number such that

$$
s_{k+1} \leq\left(1-\lambda_{k}\right) s_{k}+\lambda_{k} b_{k}+c_{k},
$$

where $\left\{\lambda_{k}\right\},\left\{b_{k}\right\}$, and $\left\{c_{k}\right\}$ satisfy the following conditions:

(1) $\sum_{k=0}^{\infty} \lambda_{k}=\infty$;

(2) either limsup $\lim _{k \rightarrow \infty} b_{k} \leq 0$ or $\sum_{k=0}^{\infty}\left|\lambda_{k} b_{k}\right|<\infty$;

(3) $c_{k} \geq 0$ for all $k$ and $\sum_{k=0}^{\infty} c_{k}<\infty$.

Then, $\lim _{k \longrightarrow \infty} s_{k}=0$.

\section{Main Results}

To introduce our iterative algorithm for solving the split feasibility problem in real Hilbert spaces, firstly, we shall assume that problem (1) is consistent, namely, its solution set, denoted by $S$, is nonempty. Secondly, we need to define a special $W$-mapping $W_{n}(n \geq 1)$ as follows:

$$
\begin{aligned}
U_{n, n+1} & =I \\
U_{n, n} & =\left(1-\eta_{n}\right) I+\eta_{n} P_{C} U_{n, n+1} \\
U_{n, n-1} & =\left(1-\eta_{n-1}\right) I+\eta_{n-1} P_{C} U_{n, n} \\
& \vdots \\
U_{n, k} & =\left(1-\eta_{k}\right) I+\eta_{k} P_{C} U_{n, k+1} \\
U_{n, k-1} & =\left(1-\eta_{k-1}\right) I+\eta_{k-1} P_{C} U_{n, k} \\
& \vdots \\
U_{n, 2} & =\left(1-\eta_{2}\right) I+\eta_{2} P_{C} U_{n, 3} \\
U_{n, 1} & =\left(1-\eta_{1}\right) I+\eta_{1} P_{C} U_{n, 2} \\
W_{n} & =U_{n, 1},
\end{aligned}
$$

where $\eta_{i}=(1 / n-i+1)(1 \leq i \leq n)$. From Lemma 4, we know that $W_{n}$ is nonexpansive.

Now, we will introduce our iterative algorithms for the split feasibility problem.

Algorithm 1. Given an initial point $x_{0} \in H_{1}$, let $\psi: H_{1} \longrightarrow H_{1}$ be a contraction mapping with $\delta \in[0,1)$. Assume that $x_{n}$ has been constructed and compute $x_{n+1}$ by the following iterative scheme:

$$
\begin{aligned}
y_{n} & =x_{n}-\tau_{n}\left[x_{n}-P_{C} x_{n}+A^{*}\left(I-P_{Q}\right) A x_{n}\right], \\
x_{n+1} & =\alpha_{n} \psi\left(x_{n}\right)+\left(1-\alpha_{n}\right) W_{n} y_{n},
\end{aligned}
$$

where $\left\{\tau_{n}\right\}$ and $\left\{\alpha_{n}\right\}$ satisfy

$$
\begin{aligned}
& \text { (1) } 0<\varepsilon \leq \tau_{n} \leq\left(2 / 1+\|A\|^{2}\right)-\varepsilon ; \\
& \text { (2) } \lim _{n \longrightarrow \infty} \alpha_{n}=0, \sum_{0}^{\infty} \alpha_{n}=\infty \text {. }
\end{aligned}
$$

Theorem 1. Let $\left\{x_{n}\right\}$ be the sequence generated by Algorithm 1, then $\left\{x_{n}\right\}$ converges strongly to a solution $x^{*}$ of the SFP (1), where $x^{*}=P_{S} \psi\left(x^{*}\right)$.

Proof. Since $\psi: H_{1} \longrightarrow H_{1}$ is a contraction mapping with $\delta \in[0,1)$ and the fact that $P_{S}$ is nonexpansive, it is clear that $P_{S} \psi: H_{1} \longrightarrow S$ is also a contraction mapping. By Banach fixed point theorem, there exists $x^{*} \in S$, such that $x^{*}=P_{S} \psi\left(x^{*}\right)$.

Since $x^{*} \in S$, that is, $x^{*} \in C$ and $A x^{*} \in Q$. By the definition of $W_{n}$, we have $x^{*}=W_{n} x^{*}$. In what follows, we will divide the proof into four steps.

Firstly, we prove that the sequence $\left\{x_{n}\right\}$ is bounded.

From (18) and Lemmas 1 and 3, we have

$$
\begin{aligned}
\left\|y_{n}-x^{*}\right\|^{2}= & \left\|x_{n}-x^{*}-\tau_{n}\left[x_{n}-P_{C} x_{n}+A^{*}\left(I-P_{Q}\right) A x_{n}\right]\right\|^{2} \\
= & \left\|x_{n}-x^{*}\right\|^{2}-2 \tau_{n}\left[\left\langle x_{n}-x^{*}, x_{n}-P_{C} x_{n}\right\rangle+\left\langle A x_{n}-P_{Q} A x_{n}, A x_{n}-A x^{*}\right\rangle\right] \\
& +\tau_{n}^{2}\left\|x_{n}-P_{C} x_{n}+A^{*}\left(I-P_{Q}\right) A x_{n}\right\|^{2}
\end{aligned}
$$


4

Journal of Mathematics

$$
\begin{aligned}
\leq & \left\|x_{n}-x^{*}\right\|^{2}-2 \tau_{n}\left(\left\|x_{n}-P_{C} x_{n}\right\|^{2}+\left\|A x_{n}-P_{Q} A x_{n}\right\|^{2}\right) \\
& +\tau_{n}^{2}\left[\left(1+\|A\|^{2}\right)\left\|x_{n}-P_{C} x_{n}\right\|^{2}+\left(1+\frac{1}{\|A\|^{2}}\right)\left\|A x_{n}-P_{Q} A x_{n}\right\|^{2}\right] \\
\leq & \left\|x_{n}-x^{*}\right\|^{2}-\tau_{n}\left[2-\tau_{n}\left(1+\|A\|^{2}\right)\right]\left(\left\|x_{n}-P_{C} x_{n}\right\|^{2}+\left\|A x_{n}-P_{Q} A x_{n}\right\|^{2}\right) .
\end{aligned}
$$

By condition (1), we have $\tau_{n}\left[2-\tau_{n}\left(1+\|A\|^{2}\right)\right]>0$, so $\left\|y_{n}-x^{*}\right\| \leq\left\|x_{n}-x^{*}\right\|$. Therefore, from (18), we obtain

$$
\begin{aligned}
\left\|x_{n+1}-x^{*}\right\| & =\left\|\alpha_{n}\left(\psi\left(x_{n}\right)-x^{*}\right)+\left(1-\alpha_{n}\right)\left(W_{n} y_{n}-x^{*}\right)\right\| \\
& \leq \alpha_{n}\left\|\psi\left(x_{n}\right)-x^{*}\right\|+\left(1-\alpha_{n}\right)\left\|W_{n} y_{n}-x^{*}\right\| \\
& \leq \alpha_{n}\left\|\psi\left(x_{n}\right)-\psi\left(x^{*}\right)\right\|+\alpha_{n}\left\|\psi\left(x^{*}\right)-x^{*}\right\|+\left(1-\alpha_{n}\right)\left\|W_{n} y_{n}-x^{*}\right\| \\
& \leq \alpha_{n} \delta\left\|x_{n}-x^{*}\right\|+\alpha_{n}\left\|\psi\left(x^{*}\right)-x^{*}\right\|+\left(1-\alpha_{n}\right)\left\|y_{n}-x^{*}\right\| \\
& \leq \alpha_{n} \delta\left\|x_{n}-x^{*}\right\|+\alpha_{n}\left\|\psi\left(x^{*}\right)-x^{*}\right\|+\left(1-\alpha_{n}\right)\left\|x_{n}-x^{*}\right\| \\
& =\left[1-\alpha_{n}(1-\delta)\right]\left\|x_{n}-x^{*}\right\|+\alpha_{n}(1-\delta) \frac{\left\|\psi\left(x^{*}\right)-x^{*}\right\|}{1-\delta} \\
& \leq \max \left\{\left\|x_{n}-x^{*}\right\|, \frac{\left\|\psi\left(x^{*}\right)-x^{*}\right\|}{1-\delta}\right\} .
\end{aligned}
$$

By introduction, we obtain

$$
\left\|x_{n+1}-x^{*}\right\| \leq \max \left\{\left\|x_{0}-x^{*}\right\|, \frac{\left\|\psi\left(x^{*}\right)-x^{*}\right\|}{1-\delta}\right\},
$$

for all $n \geq 0$. The above inequality implies that the sequence $\left\{x_{n}\right\}$ is bounded. Combining with (18), we know that $\left\{y_{n}\right\}$, $\left\{W_{n} y_{n}\right\}$, and $\left\{\psi\left(x_{n}\right)\right\}$ are also bounded.

Secondly, we show that the following inequality holds:

$$
\left\|x_{n+1}-x^{*}\right\|^{2} \leq\left(1-\tilde{\alpha}_{n}\right)\left\|x_{n}-x^{*}\right\|^{2}+\tilde{\alpha}_{n} \delta_{n},
$$

where $\tilde{\alpha}_{n}=\alpha_{n}\left(1-\delta^{2}\right)$ and

$$
\delta_{n}=\frac{2 \alpha_{n}\left\langle\psi\left(x^{*}\right)-x^{*}, x_{n+1}-x^{*}\right\rangle-t_{n}\left(\left\|x_{n}-P_{C} x_{n}\right\|^{2}+\left\|A x_{n}-P_{Q} A x_{n}\right\|^{2}\right)}{\alpha_{n}\left(1-\delta^{2}\right)},
$$

with $t_{n}=\tau_{n}\left(1-\alpha_{n}\right)\left[2-\tau_{n}\left(1+\|A\|^{2}\right)\right]$.

From equations (18) and (19) and Lemma 3, we have

$$
\begin{aligned}
\left\|x_{n+1}-x^{*}\right\|^{2}= & \left\|\alpha_{n}\left(\psi\left(x_{n}\right)-\psi\left(x^{*}\right)\right)+\left(1-\alpha_{n}\right)\left(W_{n} y_{n}-x^{*}\right)+\alpha_{n}\left(\psi\left(x^{*}\right)-x^{*}\right)\right\|^{2} \\
\leq & \left\|\alpha_{n}\left(\psi\left(x_{n}\right)-\psi\left(x^{*}\right)\right)+\left(1-\alpha_{n}\right)\left(W_{n} y_{n}-x^{*}\right)\right\|^{2}+2 \alpha_{n}\left\langle\psi\left(x^{*}\right)-x^{*}, x_{n+1}-x^{*}\right\rangle \\
\leq & \alpha_{n}\left\|\psi\left(x_{n}\right)-\psi\left(x^{*}\right)\right\|^{2}+\left(1-\alpha_{n}\right)\left\|W_{n} y_{n}-x^{*}\right\|^{2}+2 \alpha_{n}\left\langle\psi\left(x^{*}\right)-x^{*}, x_{n+1}-x^{*}\right\rangle \\
\leq & \alpha_{n} \delta^{2}\left\|x_{n}-x^{*}\right\|^{2}+\left(1-\alpha_{n}\right)\left\|y_{n}-x^{*}\right\|^{2}+2 \alpha_{n}\left\langle\psi\left(x^{*}\right)-x^{*}, x_{n+1}-x^{*}\right\rangle \\
\leq & \alpha_{n} \delta^{2}\left\|x_{n}-x^{*}\right\|^{2}+\left(1-\alpha_{n}\right)\left\|x_{n}-x^{*}\right\|^{2}-\tau_{n}\left(1-\alpha_{n}\right)\left[2-\tau_{n}\left(1+\|A\|^{2}\right)\right] \\
& \times\left(\left\|x_{n}-P_{C} x_{n}\right\|^{2}+\left\|A x_{n}-P_{Q} A x_{n}\right\|^{2}\right)+2 \alpha_{n}\left\langle\psi\left(x^{*}\right)-x^{*}, x_{n+1}-x^{*}\right\rangle \\
= & {\left[1-\alpha_{n}\left(1-\delta^{2}\right)\right]\left\|x_{n}-x^{*}\right\|^{2}+\alpha_{n}\left(1-\delta^{2}\right) } \\
& {\left[\frac{2\left\langle\psi\left(x^{*}\right)-x^{*}, x_{n+1}-x^{*}\right\rangle}{1-\delta^{2}}-\frac{\tau_{n}\left(1-\alpha_{n}\right)\left[2-\tau_{n}\left(1+\|A\|^{2}\right)\right]\left(\left\|x_{n}-P_{C} x_{n}\right\|^{2}+\left\|A x_{n}-P_{Q} A x_{n}\right\|^{2}\right)}{\alpha_{n}\left(1-\delta^{2}\right)}\right] . }
\end{aligned}
$$


So, inequality (22) holds.

Thirdly, we show that limsup $\lim _{n \rightarrow \infty} \delta_{n}$ is finite.

Since $\left\{x_{n}\right\}$ is bounded, we have

$$
\begin{aligned}
\delta_{n} & \leq \frac{2\left\langle\psi\left(x^{*}\right)-x^{*}, x_{n+1}-x^{*}\right\rangle}{1-\delta^{2}} \\
& \leq \frac{2\left\|\psi\left(x^{*}\right)-x^{*}\right\| \cdot\left\|x_{n+1}-x^{*}\right\|}{1-\delta^{2}}<\infty
\end{aligned}
$$

This implies that $\limsup _{n \longrightarrow \infty} \delta_{n}<\infty$. Next, we will show that $\lim \sup _{n \longrightarrow \infty} \delta_{n}<-1$ by contraction. If we assume that $\limsup _{n \longrightarrow \infty} \delta_{n}<-1$, then there exists $n_{0} \in N$, such that $\delta_{n} \leq-1$ for all $n \geq n_{0}$. From (22), we obtain

$$
\begin{aligned}
\left\|x_{n+1}-x^{*}\right\|^{2} & \leq\left(1-\tilde{\alpha}_{n}\right)\left\|x_{n}-x^{*}\right\|^{2}+\tilde{\alpha}_{n} \delta_{n} \\
& \leq\left(1-\tilde{\alpha}_{n}\right)\left\|x_{n}-x^{*}\right\|^{2}-\tilde{\alpha}_{n} \\
& \leq\left\|x_{n}-x^{*}\right\|^{2}-\tilde{\alpha}_{n} .
\end{aligned}
$$

By introduction, we have

$$
\left\|x_{n+1}-x^{*}\right\|^{2} \leq\left\|x_{n_{0}}-x^{*}\right\|^{2}-\sum_{i=n_{0}}^{n} \tilde{\alpha}_{i}
$$

Since $\sum_{i=0}^{\infty} \alpha_{i}=\infty, \sum_{i=0}^{\infty} \tilde{\alpha}_{i}=\left(1-\delta^{2}\right) \sum_{i=0}^{\infty} \alpha_{i}=\infty$, then there exists $N>n_{0}$, such that $\sum_{i=n_{0}}^{N} \tilde{\alpha}_{i}>\left\|x_{n_{0}}-x^{*}\right\|^{2}$. Combining with the last inequality, we have

$$
\left\|x_{N+1}-x^{*}\right\|^{2} \leq\left\|x_{n_{0}}-x^{*}\right\|^{2}-\sum_{i=n_{0}}^{N} \tilde{\alpha}_{i}<0,
$$

which is contradicted with the fact that $\left\|x_{N+1}-x^{*}\right\|^{2}$ is nonnegative. Thus, $\limsup _{n \longrightarrow \infty} \delta_{n} \geq-1$. So, $\limsup _{n \longrightarrow \infty} \delta_{n}$ is finite.

Lastly, we show that $\limsup _{n \rightarrow \infty} \delta_{n} \leq 0$.

Since limsup ${ }_{n \rightarrow \infty} \delta_{n}$ is finite, there exists a subsequence $\left\{n_{k}\right\}$ such that

$$
\begin{aligned}
& \limsup _{n \rightarrow \infty} \delta_{n}=\lim _{k \longrightarrow \infty} \delta_{n_{k}}=\lim _{k \rightarrow \infty} \frac{2 \alpha_{n_{k}}\left\langle\psi\left(x^{*}\right)-x^{*}, x_{n_{k}+1}-x^{*}\right\rangle}{\alpha_{n_{k}}\left(1-\delta^{2}\right)} \\
& -\frac{t_{n_{k}}\left(\left\|x_{n_{k}}-P_{C} x_{n_{k}}\right\|^{2}+\left\|A x_{n_{k}}-P_{Q} A x_{n_{k}}\right\|^{2}\right)}{\alpha_{n_{k}}}\left(1-\delta^{2}\right) .
\end{aligned}
$$

Since $\left\langle\psi\left(x^{*}\right)-x^{*}, x_{n_{k}+1}-x^{*}\right\rangle$ is bounded, without loss of generality, we may assume the limit of $\left\langle\psi\left(x^{*}\right)-x^{*}\right.$, $\left.x_{n_{k}+1}-x^{*}\right\rangle$ exists. From (29), we may also assume the following limit exists:

$$
\lim _{k \rightarrow \infty} \frac{t_{n_{k}}\left(\left\|x_{n_{k}}-P_{C} x_{n_{k}}\right\|^{2}+\left\|A x_{n_{k}}-P_{Q} A x_{n_{k}}\right\|^{2}\right)}{\alpha_{n_{k}}\left(1-\delta^{2}\right)} .
$$

These conditions $\lim _{n \longrightarrow \infty} \alpha_{n}=0,0<\varepsilon \leq \tau_{n_{k}} \leq(2 / 1+$ $\left.\|A\|^{2}\right)-\varepsilon$ and $t_{n}=\tau_{n}\left(1-\alpha_{n}\right)\left[2-\tau_{n}\left(1+\|A\|^{2}\right)\right]$ imply $\left(t_{n_{k}} / \alpha_{n_{k}}\left(1-\delta^{2}\right)\right) \longrightarrow \infty(k \longrightarrow \infty)$. So, we obtain

$$
\lim _{k \longrightarrow \infty}\left\|x_{n_{k}}-P_{C} x_{n_{k}}\right\|^{2}+\left\|A x_{n_{k}}-P_{Q} A x_{n_{k}}\right\|^{2}=0,
$$

that is,

$$
\lim _{k \longrightarrow \infty}\left\|x_{n_{k}}-P_{C} x_{n_{k}}\right\|=\lim _{k \longrightarrow \infty}\left\|A x_{n_{k}}-P_{Q} A x_{n_{k}}\right\|^{2}=0 .
$$

Next, we prove that any weak cluster point of the sequence $\left\{x_{n_{k}}\right\}$ is a solution of the SFP (1).

Since $\left\{x_{n_{k}}\right\}$ is bounded, let $\bar{x}$ be a weak cluster point of the sequence $\left\{x_{n_{k}}\right\}$; without loss of generality, we assume that $x_{n_{k}} \rightarrow \bar{x}$; then, we obtain $A x_{n_{k}} \rightarrow A \bar{x}$. From the fact that $P_{C}$ and $P_{Q}$ are nonexpansive, Lemma 2 implies $I-P_{C}$ and $I-P_{Q}$ are demiclosed at zero; from (32), we obtain $\bar{x}=P_{C} \bar{x}$ and $A \bar{x}=P_{Q} A \bar{x}$, i.e., $\bar{x} \in C, A \bar{x} \in Q$, hence $\bar{x} \in S$.

Finally, we show that $\left\|x_{n_{k+1}}-x_{n_{k}}\right\| \longrightarrow 0(k \longrightarrow \infty)$.

From (18) and the definition of $W_{n}$, we know

$$
\begin{aligned}
\left\|W_{n_{k}}-y_{n_{k}}\right\| & =\left\|\left(1-\eta_{1}\right) y_{n_{k}}+\eta_{1} P_{C} U_{n_{k}, 2} y_{n_{k}}-y_{n_{k}}\right\| \\
& \leq \eta_{1}\left\|y_{n_{k}}-P_{C} U_{n_{k}, 2} y_{n_{k}}\right\| \\
& =\frac{\left\|y_{n_{k}}-P_{C} U_{n_{k}, 2} y_{n_{k}}\right\|}{n_{k}} \longrightarrow 0, \quad(k \longrightarrow \infty),
\end{aligned}
$$

and, from (32), we have

$$
\begin{aligned}
\left\|y_{n_{k}}-x_{n_{k}}\right\| & =\tau_{n_{k}}\left\|x_{n_{k}}-P_{C} x_{n_{k}}+A^{*}\left(I-P_{Q}\right) A x_{n_{k}}\right\| \\
& \leq \tau_{n_{k}}\left\|x_{n_{k}}-P_{C} x_{n_{k}}\right\|+\|A\| \cdot\left\|A x_{n_{k}}-P_{Q} A x_{n_{k}}\right\| \\
& \longrightarrow 0, \quad(k \longrightarrow \infty) .
\end{aligned}
$$

So,

$$
\begin{aligned}
\left\|x_{n_{k+1}}-x_{n_{k}}\right\|= & \left\|\alpha_{n_{k}} \psi\left(x_{n_{k}}\right)+\left(1-\alpha_{n_{k}}\right) W_{n_{k}} y_{n_{k}}-x_{n_{k}}\right\| \\
\leq & \alpha_{n_{k}}\left\|\psi\left(x_{n_{k}}\right)-x_{n_{k}}\right\|+\left(1-\alpha_{n_{k}}\right)\left\|W_{n_{k}} y_{n_{k}}-x_{n_{k}}\right\| \\
& +\left(1-\alpha_{n_{k}}\right)\left\|y_{n_{k}}-x_{n_{k}}\right\| \longrightarrow 0, \quad(k \longrightarrow \infty) .
\end{aligned}
$$

This implies that any weak cluster point of $\left\{x_{n_{k+1}}\right\}$ also belongs to $S$. Without loss of generality, we assume that $\left\{x_{n_{k+1}}\right\}$ converges weakly to $\hat{x} \in S$. Now, combing (29), Lemma 1 , and the fact that $x^{*}=P_{S} \psi\left(x^{*}\right)$, we can obtain

$$
\limsup _{n \longrightarrow \infty} \delta_{n} \leq \lim _{k \longrightarrow \infty} \frac{2\left\langle\psi\left(x^{*}\right)-x^{*}, x_{n_{k+1}}-x^{*}\right\rangle}{1-\delta^{2}}=\frac{2\left\langle\psi\left(x^{*}\right)-x^{*}, \hat{x}-x^{*}\right\rangle}{1-\delta^{2}} \leq 0 .
$$


From Lemma 5, we get $\lim _{n \rightarrow \infty}\left\|x_{n}-x^{*}\right\|=0$, which ends the proof.

From Theorem 1, we obtain the following subresult on the split feasibility problem (1).

Algorithm 2. Given an initial point $x_{0} \in H_{1}$, let $u \in H_{1}$ be fixed. Assume that $x_{n}$ has been constructed and compute $x_{n+1}$ by the following iterative scheme:

$$
\begin{aligned}
y_{n} & =x_{n}-\tau_{n}\left[x_{n}-P_{C} x_{n}+A^{*}\left(I-P_{Q}\right) A x_{n}\right], \\
x_{n+1} & =\alpha_{n} u+\left(1-\alpha_{n}\right) W_{n} y_{n},
\end{aligned}
$$

where $\left\{\tau_{n}\right\}$ and $\left\{\alpha_{n}\right\}$ satisfy

(1) $0<\varepsilon \leq \tau_{n} \leq\left(2 / 1+\|A\|^{2}\right)-\varepsilon$;

(2) $\lim _{n \longrightarrow \infty} \alpha_{n}=0, \sum_{0}^{\infty} \alpha_{n}=\infty$.

Corollary 1. Let $\left\{x_{n}\right\}$ be the sequence generated by Algorithm 2, then $\left\{x_{n}\right\}$ converges strongly to a solution $x^{*}$ of the SFP (1), where $x^{*}=P_{S} u$.

\section{Data Availability}

No data were used to support this study.

\section{Conflicts of Interest}

The authors declare that they have no conflicts of interest.

\section{Authors' Contributions}

All authors contributed to each part of this work equally, and they all read and approved the final manuscript.

\section{Acknowledgments}

This research was supported by the National Natural Science Foundation of China (nos. 11601339 and 11701154), the Natural Science Foundation of the Department of Education, Henan Province (nos. 19A110020 and 20A110020), the Graduate Education Reform and Quality Improvement Project of Henan Province, Higher Education Teaching Reform and Practice Project (postgraduate education) of Henan Normal University (no. YJS2019JG01), and Program for Graduate Innovative Research of Henan Normal University (no. YL201919).

\section{References}

[1] Y. Censor and T. Elfving, "A multiprojection algorithm using bregman projections in a product space," Numerical Algorithms, vol. 8, no. 2, pp. 221-239, 1994.

[2] Y. Censor, T. Bortfeld, B. Martin, and A. Trofimov, "A unified approach for inversion problems in intensity-modulated radiation therapy," Physics in Medicine and Biology, vol. 51, no. 10, pp. 2353-2365, 2006.

[3] C. Byrne, "Iterative oblique projection onto convex sets and the split feasibility problem," Inverse Problems, vol. 18, no. 2, pp. 441-453, 2002.
[4] Z. C. Jin, C. C. Lu, Q. Q. Yang, and R. K. Zhao, "Extra-gradient methods for solving split feasibility and fixed point problems," Fixed Point Theory and Applications, vol. 192, 2015.

[5] G. López, v Matín, F. H. Wang, and H. K. Xu, "Solving the split feasibility problem without prior knowledge of martix norms," Inverse Problems, vol. 28, no. 8, Article ID 85004, 2012.

[6] A. Moudafi, "A relaxed alternating CQ-algorithm for convex feasibility problems," Nonlinear Analysis: Theory, Methods \& Applications, vol. 79, pp. 117-121, 2013.

[7] A. Moudafi, "Alternating CQ-algorithm for convex feasibility and split fixed-point problems," Journal of Nonlinear and Convex Analysis, vol. 15, pp. 809-818, 2014.

[8] F. Wang, "Strong convergence of two algorithms for the split feasibility problem in banach spaces," Optimization, vol. 67, no. 10, pp. 1649-1660, 2018.

[9] F. H. Wang and H. K. Xu, "Approximating curve and strong convergence of the CQ algorithm for the split feasibility problem," Journal of Inequalities and Applications, vol. 2010, no. 1, 13 pages, Article ID 102085, 2010.

[10] F. Wang, "Polyak's gradient method for split feasibility problem constrained by level sets," Numerical Algorithms, vol. 77, no. 3, pp. 925-938, 2018.

[11] H. Yu, W. Zhan, and F. Wang, "The ball-relaxed CQ algorithms for the split fesaibility problem," Optimization, vol. 67, no. 10, pp. 1687-1699, 2018.

[12] A. Moudafi and B. S. Thakur, "Solving proximal split feasibility problems without prior knowledge of operator norms," Optimization Letters, vol. 8, no. 7, pp. 2099-2110, 2014.

[13] Y. Shehu, G. Cai, and O. S. Iyiola, "Iterative approximation of solutions for proximal split feasibility problems," Fixed Point Theory and Applications, vol. 123, no. 1, 2015.

[14] Y. Shehu and F. U. Ogbuisi, "Convergence analysis for proximal split feasibility problems and fixed point problems," Journal of Applied Mathematics and Computing, vol. 48, no. 12, pp. 221-239, 2015.

[15] H. H. Bauschke and P. L. Combettes, Convex Analysis and Monotone Operator Theory in Hilbert Space, Springer, Berlin, Germany, 2011.

[16] K. Shimoji and W. Takahashi, "Strong convergence to common fixed points of inifinite nonexpansive mappings and applications," Taieanese Journal of Mathematics, vol. 5, no. 2, pp. 387-404, 2001.

[17] H.-K. Xu, "Iterative algorithms for nonlinear operators," Journal of the London Mathematical Society, vol. 66, no. 1, pp. 240-256, 2002. 